\title{
EURECOM \\ EURECOM
}

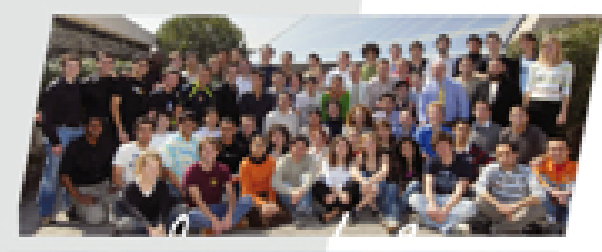

\section{The quest for multi-headed worms}

Van-Hau PHAM, Marc DACIER, Guillaume URVOY-KELLER, Taoufik EN-NAJJARY 


\section{Outline}

- Introduction

- Method and Implementation

- Experimental Environment

- Approach

- Results

- Conclusion 


\section{Definition of multi-headed worms}

- Combining several known exploits

- Only one exploit used to attack against a new target

- Less efficient to propagate but more stealthy

- Example: Welchia

(Pouget, Fabien;Urvoy-Keller, Guillaume;Dacier, Marc "Time signatures to detect multi-headed stealthy attack tools" 18th Annual FIRST Conference, June 25-30, 2006, Baltimore, USA ) 


\section{HOW TO DETECT multi-headed worms ?}

- Multi-headed worms leave correlated attack traces

Example: a multi-headed worm carries two attack vectors $A$ and $B$

- At each time-step, an infected machine makes 5 attacks

- In 2/5 times, using attack vector $A$, in $3 / 5$ times, using attack vector $B$

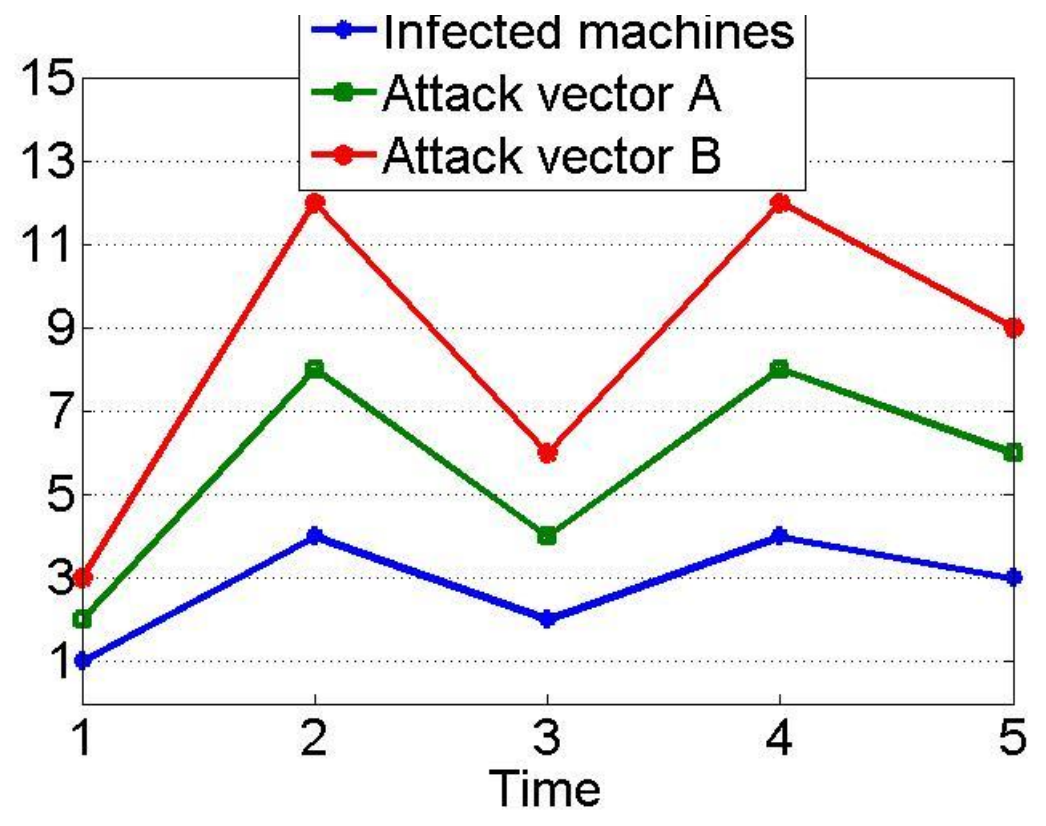

\# of attacks of $A$ and $B$ always vary together, and they are a function of \# of infected machines

- Correlation of attack traces is a sign of multi-headed worms 


\section{Shortcomings when applying to a large dataset}

- Too many attack traces

- Sliding windows vs. whole period: to deal with

- the overlapping between different activities

- the incompleteness of observation

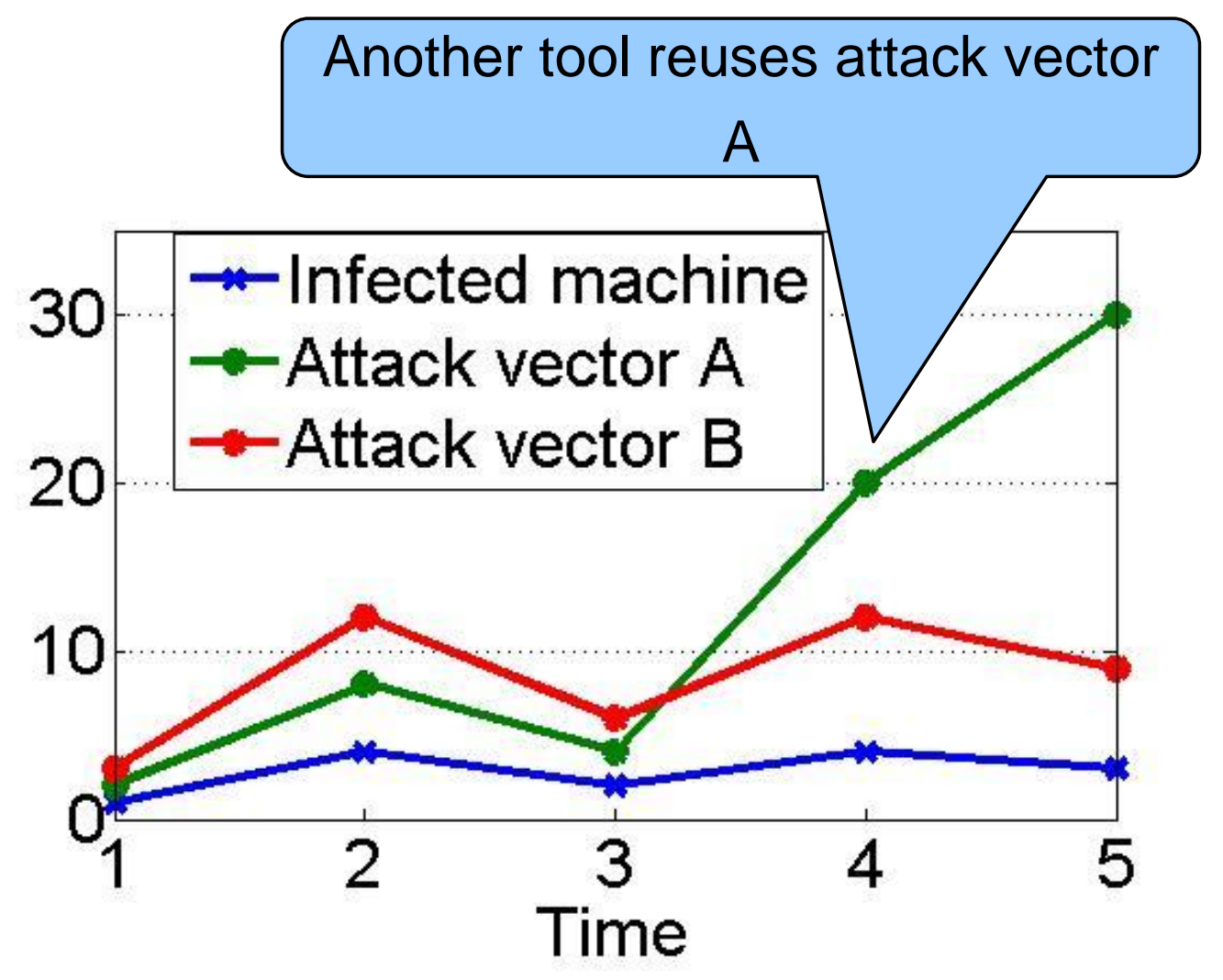

- At time $=4$, another tool reuses attack vector $\mathrm{A}$

Correlation period of trace $A$ and $B$ is from 1 to 3 


\section{Outline}

- Introduction

- Method and implementation

- Experimental Environment

- Approach

- Results

- Conclusion 


\section{Platform}

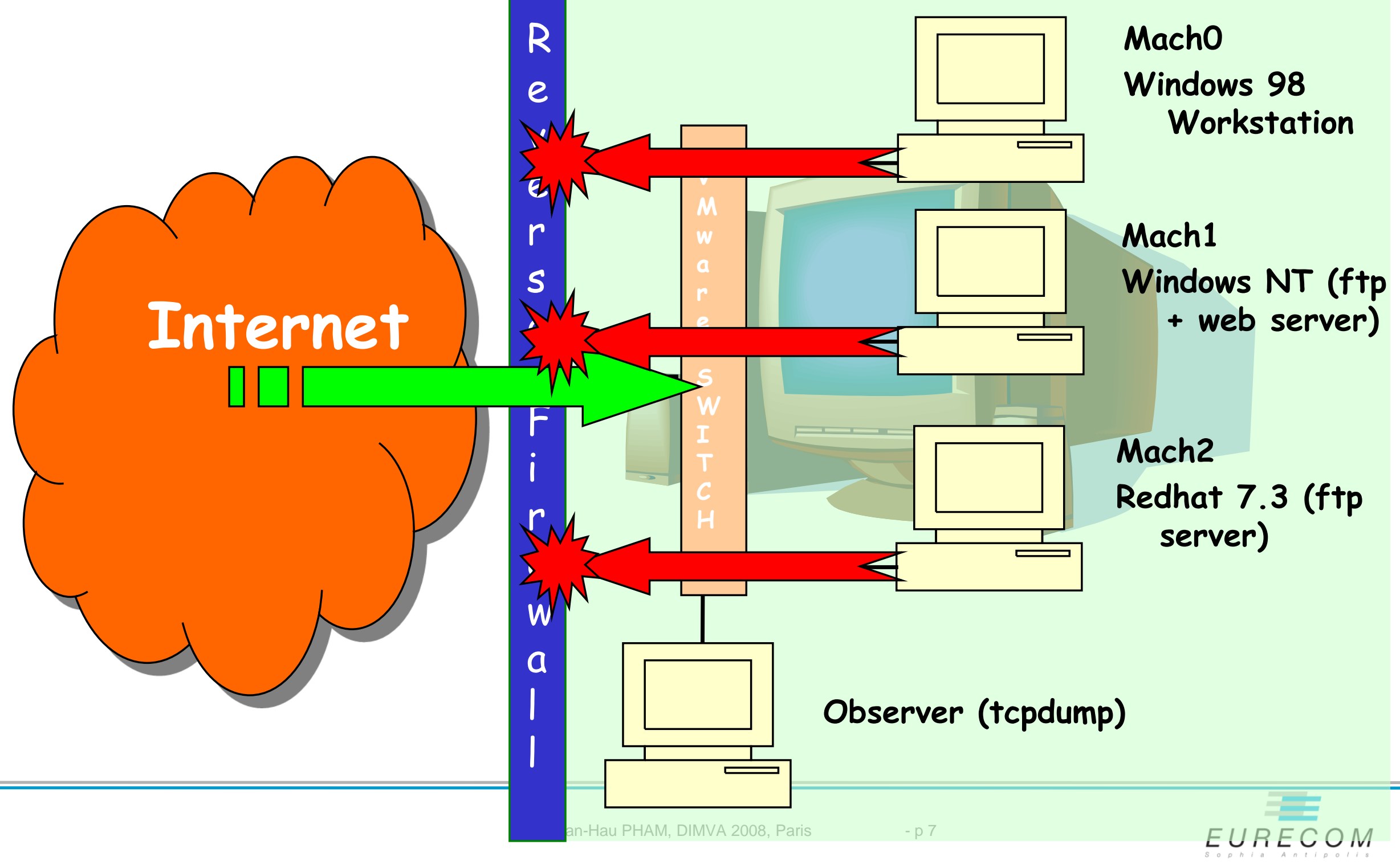




\section{Terminology}

- Cluster: attacking sources leaving similar traces on our platforms

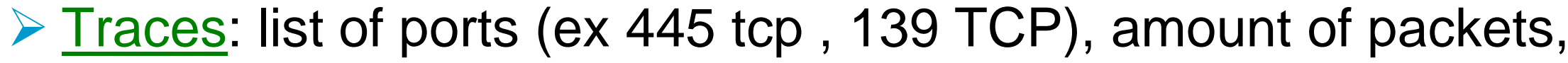
attack duration,...

- Cluster time series: amount of sources, on a daily basis, associated to a given cluster on a given platform

- Platform time series: sum of all cluster time series associated to a given platform 


\section{Dataset description}

- 15 months of data

- 28 platforms

$\square$ With the uptime rate higher than $90 \%$

- 15 countries

59,000 cluster time series,

a huge amount of data!!! 


\section{Outline}

- Introduction

- Method and Implementation

- Experimental Environment

- Approach

- Results

- Conclusion 


\section{Our approach}

- Preprocessing technique

- Reduce the number of clusters

- Correlated groups of platform time series

- Instead of correlations between clusters

- Root cause extraction

- Relate clusters time series to platform time series 


\section{Preprocessing technique}

- Cluster time series can be classified into 3 families:

- Stable time series: excluded since correlation is meaningless

- Peaked time series: trivial cases, leave for future work Stable time series

- Strongly varying time series: strongly active attack tools, kept for our analysis

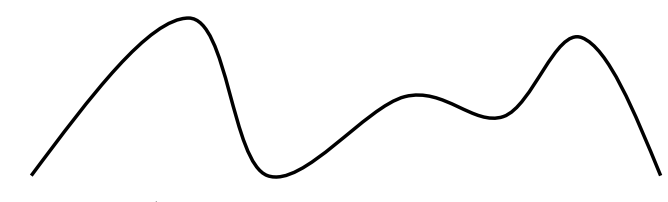

Strong varying time series 


\section{Groups of correlated platform time series (1)}

\section{$\underline{\text { Technique }}$}

- We filter out the stable and peak time series to build platform time series

- We use the sliding window to identify all periods where there exist groups of correlated platform time series

$\rightarrow \sim 28^{\wedge} 2{ }^{*}(450-30)$ instead of $\sim 59000^{\wedge} 2{ }^{*}(450-30)$ operations to compute the correlation 


\section{Groups of correlated platform time series (2)}

\section{Example}

- $\quad$ platform time series: $1,2,15$

- Period: from day 1 to day 100

- Result: correlation of platform time series 2 and 15 from day 30 to day 90
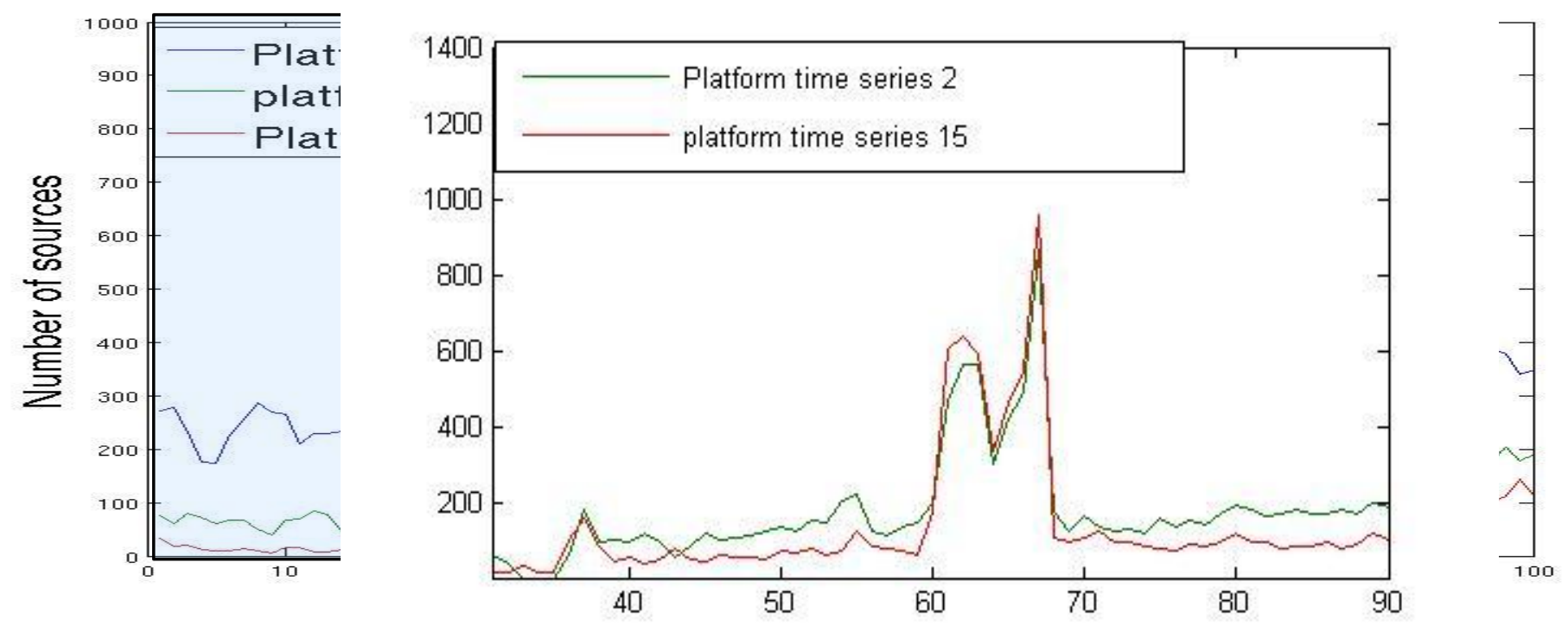


\section{Root cause extraction (1)}

- The root causes are clusters that explain the correlation of the groups of correlated platform time series

- In each correlated period, to identify them, we look for the clusters that are similar to the platform time series, platform by platform 


\section{Root cause extraction (2)}

group of correlated platform time series
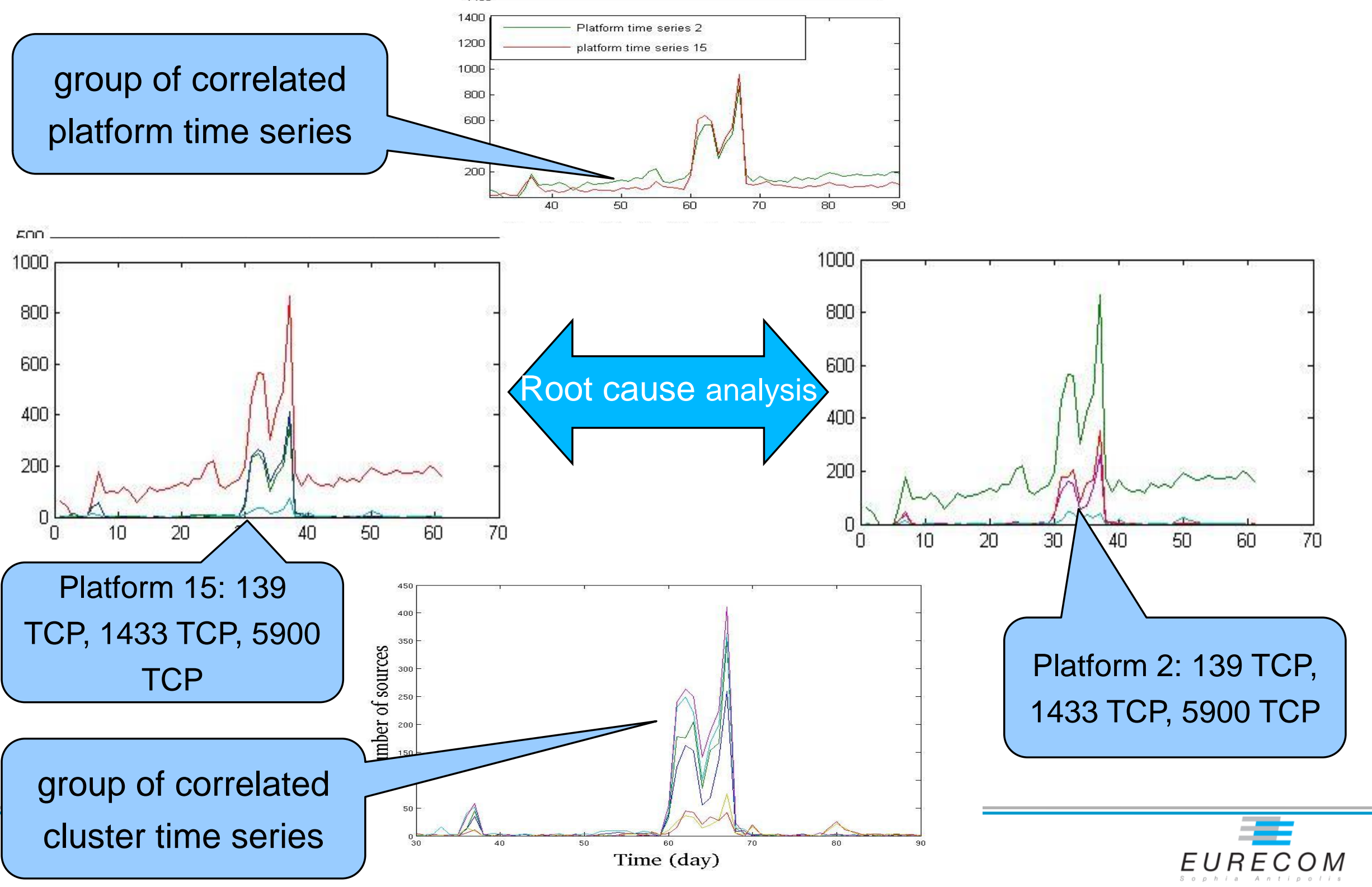


\section{Outline}

- Introduction

- Method and Implementation

- Experimental Environment

- Approach

- Results

- Conclusion 


\section{Results}

- We found out 28 correlated groups involving 130 cluster time series, which can be classified into:

- Non multi-headed worms groups (21 groups)

- Single root cause groups (10 groups)

- Multiple root causes groups (11 groups)

- Multi-headed worms (7 groups) 


\section{Single root cause (10 groups)}

- They correspond to phenomena where a single, and always the same, cluster is the root cause of the correlation of platform time series

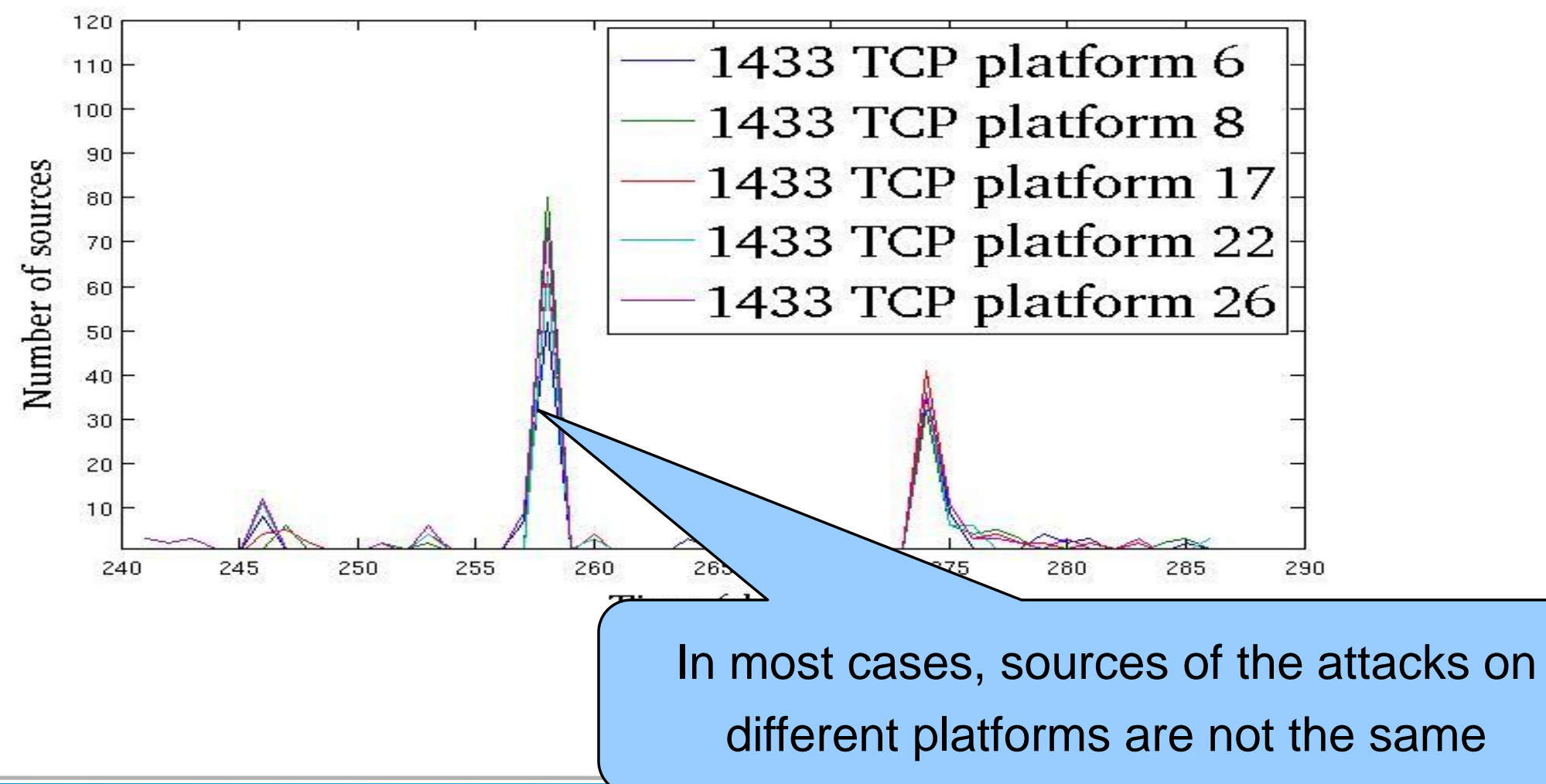




\section{Multiple root causes groups}

- Non deterministic attack tools

- Attack the same list of ports but in different orders,...

- Leave different traces $\rightarrow$ different clusters

- Fingerprinting worms leave different attack traces on different operating systems

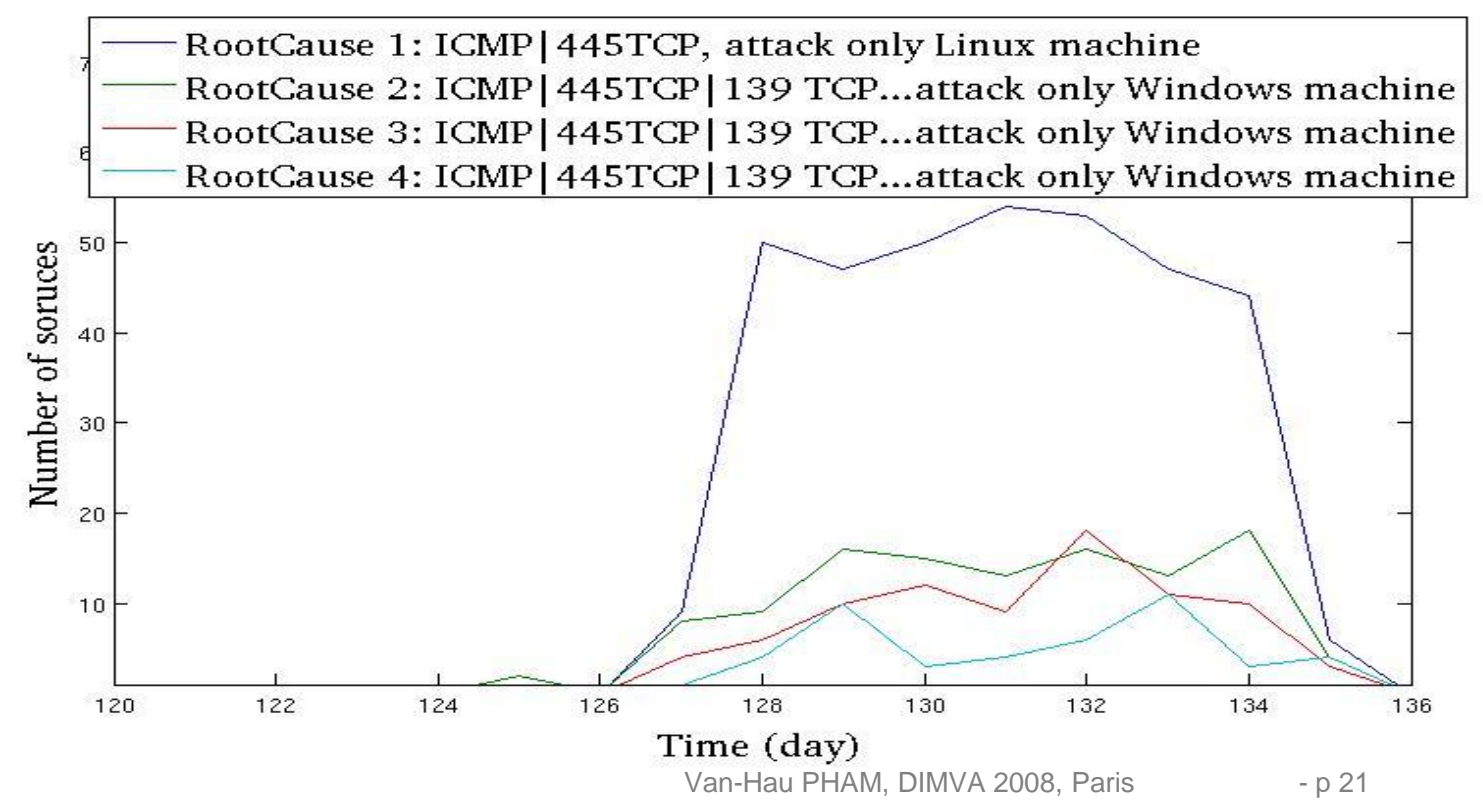




\section{Example of a multi-headed worm}

$>$ Multi-headed worms carry many attack vectors, but they use only one of them to attack a given target.

$>$ Example: A multi-headed worm, observed on two platforms 2 and 15, has three attack vectors to attack 139 TCP, 1433 TCP, and 5900 TCP

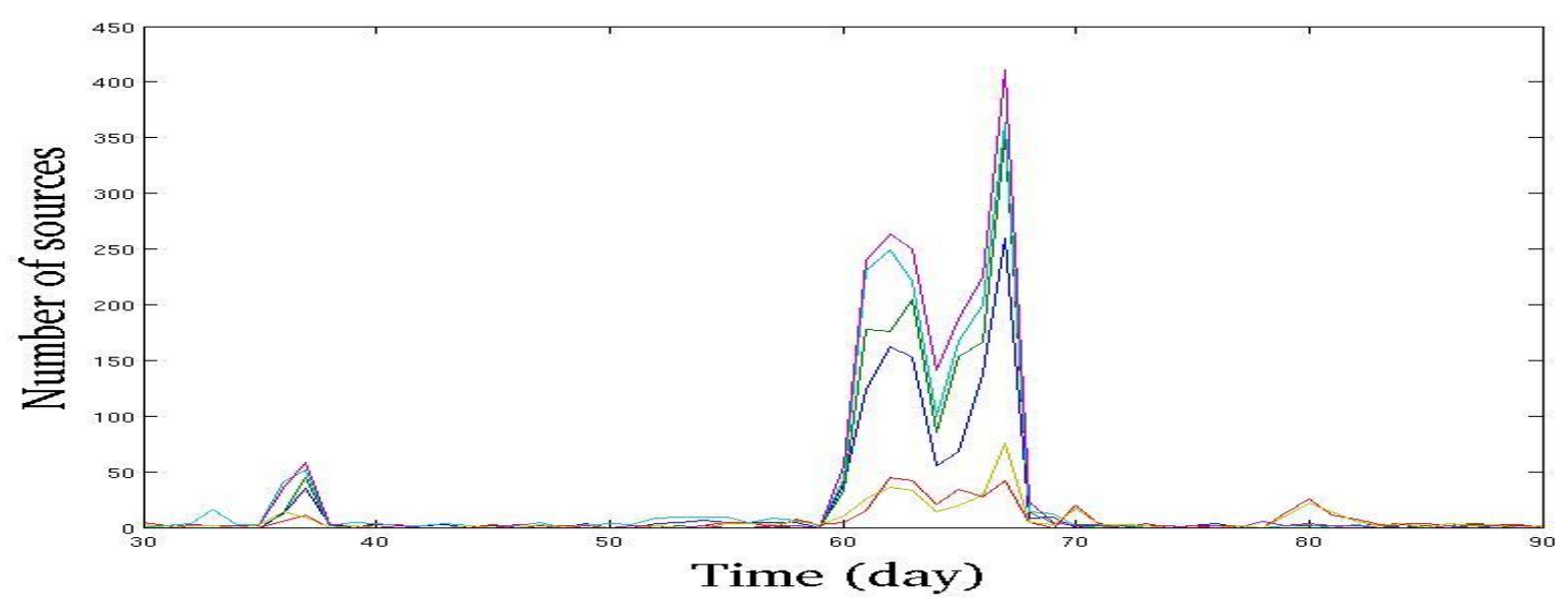




\section{Some characteristics of multi-headed worms}

- Around $60 \%$ of multi-headed worms have 2 attack vectors

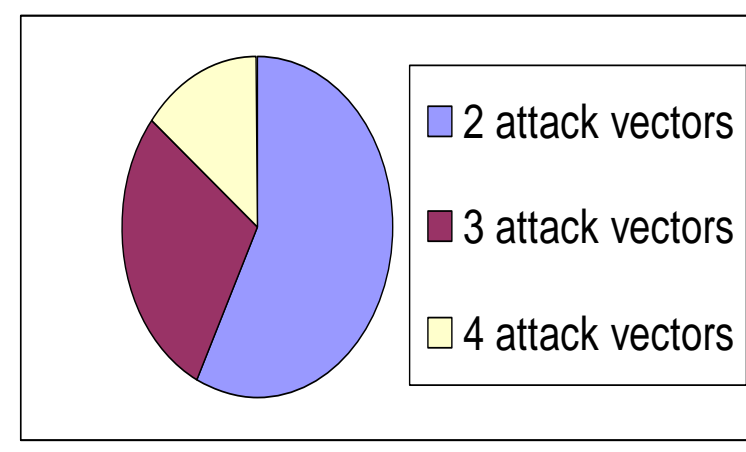

- $80 \%$ of cases, the duration of appearance is from 30 to 40 days

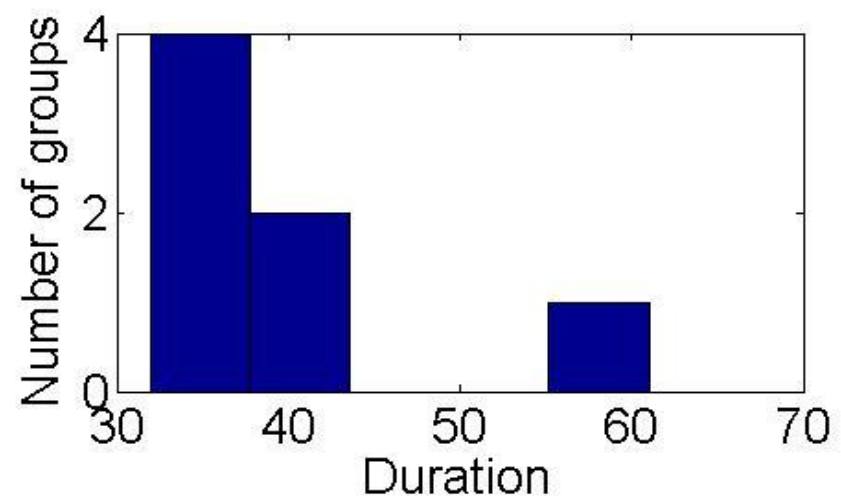

- $60 \%$ of them have been seen only on 2 platforms

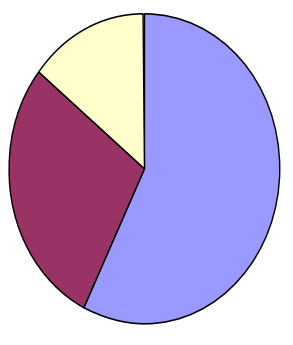

$\square 2$ platforms

$\square 3$ platforms

$\square 5$ platforms 


\section{Conclusion}

- The approach based on platform time series works and it returns not only multi-headed worms, but other interesting phenomena.

- There are not so many multi-headed worms existing in the wild, and they have the locality property, and appear only in a short period of time 


\section{Future works}

- Testing the brute-force approach on a limited amount of platforms to detect all possible correlation.

- Applying the method recursively

- Studying the peaked time series family 